\title{
Prognostic nomogram for patients with invasive endocervical adenocarcinoma incorporating HPV E6/E7 mRNA in situ hybridization and clinical characteristics
}

\section{Li-Li Liu}

Sun Yat-sen University Cancer Center https://orcid.org/0000-0002-4347-2038

\section{Shu-Lin Chen}

Sun Yat-sen University Cancer Center

\section{Shu-Mei Yan}

Sun Yat-sen University Cancer Center

Yue Li

Sun Yat-sen University Cancer Center

\section{Xia Yang}

Sun Yat-sen University Cancer Center

\section{Shi-Wen Zhang}

Sun Yat-sen University Cancer Center

\section{Hai-Xia Yang}

Sun Yat-sen University Cancer Center

\section{Ting Wan}

Sun Yat-sen University Cancer Center

\section{Rong-Zhen Luo ( $\square$ luorzh@sysucc.org.cn )}

Sun Yat-sen University Cancer Center https://orcid.org/0000-0002-6599-0370

\section{Research article}

Keywords: Endocervical adenocarcinoma, Prognosis, HPV E6/E7 mRNA in situ hybridization, Lymphovascular invasion, Lymph node involvement

Posted Date: October 7th, 2020

DOl: https://doi.org/10.21203/rs.3.rs-86110/v1

License: (9) This work is licensed under a Creative Commons Attribution 4.0 International License. Read Full License 


\section{Abstract}

Background: Human papillomavirus (HPV) E6/E7 mRNA in situ hybridization (HPV E6/E7 RNAscope) appears to be a sensitive and specific method. We aimed to examine the diagnostic and prognostic utility of this technique in endocervical adenocarcinoma (ECA) and build a useful prognostic nomogram model using this approach.

Methods: The model was constructed from a retrospective study of 200 patients with ECA who had undergone surgery at Sun Yat-sen University Cancer Center between 2010 and 2014. The model's predictive efficiency and discriminative capability were defined by a concordance index (C-index) and calibration curve.

Results: The overall sensitivity and specificity of HPV E6/E7 RNAscope for distinguishing HPV-associated adenocarcinoma (HPVA) from non HPV-associated adenocarcinoma (NHPVA) in the whole cohort were $75.8 \%$ and $80 \%$, respectively, compared to $60.2 \%$ and $90.0 \%$ for 16 immunohistochemistry, $80.5 \%$ and $80.0 \%$ for HPV DNA, and $63.3 \%$ and $50 \%$ for HPV genotype. The independent factors derived from multivariable analysis of the whole cohort to predict overall survival (OS) were age, lymphovascular invasion (LVI), lymph node involvement (LNI), and HPV E6/E7 RNAscope, which were all incorporated into the nomogram with (nomogram B) or without (nomogram A) FIGO stage and treatment. The C-index of nomogram $A$ for predicting OS was $0.825(p=0.002$ and $p<0.001)$, which was significantly higher than the $\mathrm{C}$-index for FIGO stage (0.653) and treatment (0.578). No significant difference occurred between nomograms $A(0.825)$ and $B(0.836)$. Furthermore, a risk stratification system was established to accurately stratify patients with ECA into two subgroups with significantly different prognosis.

Conclusions: HPV E6/E7 RNAscope is highly specific for ECA, and the proposed nomogram using HPV E6/E7 RNAscope showed more accurate outcome in terms of prognosis in patients with ECA.

\section{Introduction}

Endocervical adenocarcinoma (ECA) accounts for $15-20 \%$ of all cervical carcinomas. Studies have reported that ECA is increasing in incidence, and that it often occurs in young women(1). Recent studies have reported that the 5 -year overall survival (OS) rate is $10-20 \%$ lower in ECA than in squamous cell carcinoma(2). Additionally, the International Federation of Gynecology and Obstetrics (FIGO) staging system is the gold standard for predicting outcomes in patients with ECA, and concurrent chemoradiotherapy is the standard treatment for advanced ECA. However, patients with ECA that have the same FIGO stage can have marked heterogeneities of outcome $(3,4)$. According to the latest World Health Organization (WHO) Classification of Tumors of Female Reproductive Organs, the most frequent type of ECA is usual-type, which is human papillomavirus (HPV)-related(5). A new classification-the International Endocervical Adenocarcinoma Criteria and Classification (IECC)-categorizes endocervical adenocarcinomas (ECAs) based on morphological features linked to etiology (i.e., HPV infection), resulting in the separation of ECAs into HPV-associated (HPVA) and non-HPVA (NHPVA) types(4). 
Comparing HPVA with NHPVA types reveals essential differences in tumor behavior and patient survival, with significantly worse clinical outcomes for patients with NHPVA-type tumors $(6,7)$. It follows that a classification based on pathogenesis and other clinicopathological factors may be more clinically informative and reproducible than the current FIGO scheme. Hence, to complement the FIGO staging system, researchers must ascertain better prognostic determinants.

The validity of this new classification is supported by HPV status and, at present, by limited clinical data $(8,9)$. In the present study, the following HPV tests were used: PCR, genotype, RNAscope, and immunochemistry (IHC) against p16 protein. Plasma HPV DNA levels can be determined using PCR, providing a potential marker for cervical cancer(10). The other assays can be conducted on formalinfixed, paraffin-embedded (FFPE) samples. Immunostaining for p16 is a cost-effective marker of viral infection(11). It is highly sensitive, but lacks specificity(11). PCR for HPV genotyping is the gold-standard assay to diagnose active HPV infection(12). Chromogenic in situ hybridization against RNA can identify HPV in situ via microscopic observation(13). The technique is expensive, but can be used on FFPE samples(14). In situ detection of HPV E6/E7 mRNA using the RNAscope also appears to be a sensitive and specific method, and the present study aimed to investigate the potential prognostic utility of this technique in ECAs.

Numerous disagreements have arisen regarding the traditional staging system, which is completely based on the anatomical data. By stratifying pattern C ECAs into subgroups based on LVI and LNI status, clinicians could better determine treatment in patients with pattern $C$ tumors(15). As such, it may be suboptimal to stratify patients based on a single biomarker, such as lymphovascular invasion (LVI) or lymph node involvement (LNI) alone, because it can lead to misclassification of tumors and subsequent misallocation of patients with an undesirable prognosis. The combination of LVI and LNI status has shown better prognostic value(15). However, no currently available nomogram model for patients with ECA combines HPV, LVI, and LNI status, and only some of these parameters have been assessed in these patients.

Hence, in the present study, we built a model that included HPV E6/E7 RNAscope and clinicopathological variables to predict overall survival (OS) in patients with ECA. Moreover, we conducted an examination to compare the predictive capability of the nomogram with those of HPV E6/E7 RNAscope alone and current popular staging systems. Portentous survival prediction models that follow the FIGO staging system are insufficient. Hence, we built a nomogram for predicting OS in patients with ECA.

\section{Materials \& Methods}

\section{Patients and samples included}

In the present retrospective study included 200 patients with pathologically diagnosed ECA who had been treated at the Sun Yat-sen University Cancer Center between January 2010 and December 2014. The inclusion criteria were as follows: (1) pathological diagnosis of ECA, and (2) complete clinical data and laboratory tests results. The exclusion criteria were as follows: (1) systemic metastasis with other 
confirmed malignancies, and (2) previous cancer treatment. The last follow-up was performed in June 2020. The histological diagnoses were based on our proposal for a new ECA classification: the IECC 2017(9).

\section{Immunohistochemistry (IHC)}

A tissue microarray consisting of the ECA and adjacent non-tumorous tissue was constructed using a tissue array instrument (Minicoreexcilone; Minicore, UK). For each patient, hematoxylin and eosin (H\&E) stained slides were examined, and at least two areas from different regions were marked for sampling. Each tissue core with a diameter of $1.0 \mathrm{~mm}$ was punched from the marked fields and re-embedded. FFPE ECA sections were dewaxed in xylene and graded alcohols, hydrated, and washed in PBS. After pretreatment in a microwave oven, endogenous peroxidase was blocked using $3 \%$ hydrogen peroxide in methanol for 20 minutes. This was followed by avidin-biotin blocking using a kit (DAKO, Germany). The slides were then incubated with antibodies for p16 (Roche, Germany), MLH1 (Roche (M1), Germany), PMS2 (Dako [EP51], Germany), MSH2 (ZA0622, Zhongshan, China), MSH6 (Roche [SP93], Germany), and $\mathrm{Ki}-67$ (ZA0502, Zhongshan, China) overnight at $4{ }^{\circ} \mathrm{C}$, washed in PBS, and incubated with biotinylated goat anti-rabbit/mouse antibodies (DAKO, Germany). The slides were developed using diaminobenzidine (DAB) and counterstained with hematoxylin. Two experienced pathologists evaluated the staining; p16 was interpreted as positive when diffuse, block-like staining was found in all cores; no staining or patchy staining was interpreted as unfavorable. MSH2/MSH6/PMS2/MLH1 was interpreted as positive if $\geq 1 \%$ of the tumor cell nuclei were positive. Representative images were show in Fig. S1.

\section{Real-time PCR for HPV DNA}

HPV DNA viral load were routinely evaluated using quantitative PCR before therapy, as described in previous studies $(16,17)$.

\section{HPV Genotype}

Tumor tissue for PCR testing was removed in tumors that were not represented in tissue microarrays, as described in a previous study(18). The Roche Cobas 4800 system (Roche, Pleasanton, CA) was used to detect HPV; it evaluates the presence of the following 14 types of HPV DNA: 16, 18, 31, 33, 35, 39, 45, 51, $52,56,58,59,66$, and 68.

\section{HPV E6/E7 MRNA in situ hybridization}

The stained slides of each specimen were examined using the RNAscope scoring system, as described in previous studies $(19,20)$. High-risk HPV subtypes were evaluated on all ECAs in the tissue microarray that had sufficient tissue to allow scoring $(n=200)$. HPV fluorescent in situ hybridization $(F I S H)$ was performed using a chromogen and the RNAscope system (Advanced Cell Diagnostics; catalog No.

312598). The RNAscope probe "HPV HR18" contains probes that target E6 and E7 mRNA in the following high-risk subtypes: HPV16, 18, 26, 31, 33, 35, 39, 45, 51, 52, 53, 56, 58, 59, 66, 68, 73, and 82. RNAscope results were classified into five degrees based on the following scoring guidelines: score 0 , no staining or less than one dot in every ten cells (visible at 40x magnification); score 1, 1-3 dots per cell (visible at 2040x magnification); score 2, 4-10 dots per cell, with very few dot clusters (visible at 20-40x 
magnification); score $3,>10$ dots per cell, with $<10 \%$ of positive cells having dot clusters (visible at $20 x$ magnification); score $4,>10$ dots per cell with $>10 \%$ of positive cells having dot clusters (visible at $20 x$ magnification). Cases with RNAscope score $\geq 1$ were identified as positive.

\section{Cut-off values of variables}

Among the patients enrolled, the following variables were recorded: age, treatment (Surgery with or without chemotherapy/radiotherapy), HPV DNA, HPV subtype, HPV E6/E7 RNAscope, histological type, tumor size, differentiation, $\mathrm{LVI}$, invasion level of the uterine cervix, $\mathrm{LNI}$, parametrium invasion, surgical margin, MMR status, p16, Ki-67, and FIGO stage. Specifically, 1 pg/mL of serum HPV DNA levels, as detected by PCR, was determined as the cutoff level. The best cutoff values were determined using Xtile(21), with the following results: age, 37 years; RNAscope, 3.3 (Fig. S2A); tumor size, $4.5 \mathrm{~cm}$; and Ki-67 (12.5\%).

\section{Statistical Analysis}

Statistical investigations were conducted using SPSS 19.0 (Chicago, IL, USA) and R 3.4.0 (http://www. Rproject.org/). Survival analysis was conducted utilizing the Kaplan-Meier examination. Statistical analyses were significant if the two-sided $p$-value was $<0.05$.

\section{Results}

\section{Characteristics and survival in patients enrolled}

We enrolled 200 patients with ECA for analysis, comprising 185 with HPVA and 15 with NHPVA (Table 1). Across both groups, the median follow-up period was 68 months (range: 1.5-125.1 months). Moreover, the 1-, 3-, and 5-year OS rates were $99.5 \%, 90.5 \%, 85.0 \%$, respectively. 
Table 1. Patient demographics and clinical charateristics.

\begin{tabular}{|c|c|c|c|c|}
\hline \multirow[t]{2}{*}{ Characteristic } & Total $(n=200)$ & HPVA $(n=186)$ & NHPVA $(n=16)$ & \multirow[b]{2}{*}{$P^{A}$} \\
\hline & No. (\%) & No. (\%) & No. (\%) & \\
\hline Age & & & & 0.014 \\
\hline$\leq 37$ & $34(17.0 \%)$ & $28(82.4 \%)$ & $6(17.6 \%)$ & \\
\hline$>37$ & $166(83.0 \%)$ & $157(94.6 \%)$ & $9(5.4 \%)$ & \\
\hline Serum HPV DNA & & & & 0.000 \\
\hline Negative $(0-1 \mathrm{pg} / \mathrm{ml})$ & $34(17.0 \%)$ & $26(76.5 \%)$ & $8(23.5 \%)$ & \\
\hline Positive $(\geq 1 \mathrm{pg} / \mathrm{ml})$ & $110(55.0 \%)$ & $108(98.2 \%)$ & $2(1.8 \%)$ & \\
\hline Not avaiable & $56(28.0 \%)$ & $51(51.8 \%)$ & $5(4.2 \%)$ & \\
\hline HPV genotype & & & & 0.273 \\
\hline HPV 16 & $49(24.5 \%)$ & $47(95.9 \%)$ & $2(4.1 \%)$ & \\
\hline HPV 18 & $60(30.0 \%)$ & $57(95.5 \%)$ & $3(5.0 \%)$ & \\
\hline Other & $11(5.5 \%)$ & $11(100.0 \%)$ & $0(0.0 \%)$ & \\
\hline Negative & $72(36.0 \%)$ & $63(87.5 \%)$ & $9(12.5 \%)$ & \\
\hline Not avaiable & $8(4 \%)$ & $7(87.5 \%)$ & $1(12.5 \%)$ & \\
\hline HPV RNAscope & & & & 0.003 \\
\hline$\leq 3.3$ & $99(49.5 \%)$ & $86(46.5 \%)$ & $13(13.1 \%)$ & \\
\hline$>3.3$ & $101(50.5 \%)$ & $99(98.0 \%)$ & $2(2.0 \%)$ & \\
\hline Tumor size (cm) & & & & 0.004 \\
\hline$\leq 4.5$ & $178(89.0 \%)$ & $168(94.4 \%)$ & $10(5.6 \%)$ & \\
\hline$>4.5$ & $22(11.0 \%)$ & $17(77.3 \%)$ & $5(22.7 \%)$ & \\
\hline FIGO stage & & & & 0.000 \\
\hline $\mathrm{I}$ & $141(70.5 \%)$ & $137(97.2 \%)$ & $4(2.8 \%)$ & \\
\hline II & $51(25.5 \%)$ & $43(84.3 \%)$ & $8(15.7 \%)$ & \\
\hline III & $5(2.5 \%)$ & $4(80.0 \%)$ & $1(20.0 \%)$ & \\
\hline IV & $3(1.5 \%)$ & $1(33.3 \%)$ & $2(66.7 \%)$ & \\
\hline Differentiation & & & & 0.537 \\
\hline Good & $10(5.0 \%)$ & $7(70.0 \%)$ & $3(30.0 \%)$ & \\
\hline Moderate & $106(53.0 \%)$ & $96(90.6 \%)$ & $10(9.4 \%)$ & \\
\hline Poor & $84(42.0 \%)$ & $82(97.6 \%)$ & $2(2.4 \%)$ & \\
\hline LVI & & & & 0.141 \\
\hline None (0) & $138(69.0 \%)$ & $130(94.2 \%)$ & $8(5.8 \%)$ & \\
\hline Focal (1-4) & $37(18.5 \%)$ & $31(83.8 \%)$ & $6(16.2 \%)$ & \\
\hline Moderate (5-9) & $15(7.5 \%)$ & $14(93.3 \%)$ & $1(6.7 \%)$ & \\
\hline Extensive $(\geq 10)$ & $10(66.7 \%)$ & $10(100.0 \%)$ & $0(0.0 \%)$ & \\
\hline Invasion level of uterine cervix & & & & 0.008 \\
\hline $\begin{array}{l}<1 / 3 \\
1 / 3-2 / 3\end{array}$ & $\begin{array}{l}54(27.0 \%) \\
61(31.0 \%)\end{array}$ & $\begin{array}{l}53(98.1 \%) \\
60(96.8 \%)\end{array}$ & $\begin{array}{l}1(1.9 \%) \\
2(3.2 \%)\end{array}$ & \\
\hline$\geq 2 / 3$ & $84(42.0 \%)$ & $72(85.7 \%)$ & $12(14.3 \%)$ & \\
\hline Lymph nodes invasion & & & & 0.024 \\
\hline No & $154(77.0 \%)$ & $146(94.8 \%)$ & $8(5.2 \%)$ & \\
\hline Yes & $46(23.0 \%)$ & $39(84.8 \%)$ & $7(46.7 \%)$ & \\
\hline Parametrium invasion & & & & 0.599 \\
\hline No & $181(90.5 \%)$ & $168(92.8 \%)$ & $13(7.2 \%)$ & \\
\hline Yes & $19(9.5 \%)$ & $17(89.5 \%)$ & $2(10.5 \%)$ & \\
\hline Surgical margin & & & & 0.000 \\
\hline No & $187(93.5 \%)$ & $177(94.7 \%)$ & $10(5.3 \%)$ & \\
\hline Yes & $13(6.5 \%)$ & $8(61.5 \%)$ & $5(38.5 \%)$ & \\
\hline Treatment & & & & 0.235 \\
\hline
\end{tabular}




\begin{tabular}{lllll} 
Surgery & $83(41.5 \%)$ & $80(96.4 \%)$ & $3(3.6 \%)$ & \\
\hline Surgery+chemotherapy & $21(10.5 \%)$ & $20(95.2 \%)$ & $1(4.8 \%)$ & \\
\hline Surgery+radiotherapy & $41(20.5 \%)$ & $36(87.8 \%)$ & $5(12.2 \%)$ & \\
\hline Surgery+chemoradiation & $55(27.5 \%)$ & $49(89.1 \%)$ & $6(10.9 \%)$ & 0.978 \\
MMR status & & & & \\
\hline dMMR & $13(6.5 \%)$ & $12(92.3 \%)$ & $1(7.7 \%)$ & \\
\hline pMMR & $187(93.5 \%)$ & $173(92.5 \%)$ & $14(7.5 \%)$ & 0.001 \\
\hline P16 IHC & & & & \\
\hline Negative & $82(41.0 \%)$ & $70(85.4 \%)$ & $12(14.6 \%)$ & \\
\hline Positive & $118(59.0 \%)$ & $115(97.5 \%)$ & $3(2.5 \%)$ & 0.131 \\
\hline Ki-67 IHC & & & & \\
\hline$\leq 12.5 \%$ & $48(24 \%)$ & $42(87.5 \%)$ & $6(12.5 \%)$ & \\
\hline$>12.5 \%$ & $152(76 \%)$ & $143(94.1 \%)$ & $9(5.9 \%)$ & \\
\hline
\end{tabular}

${ }^{a}$ Chi-square test. HPVA, HPV-associated adenocarcinoma; NHPVA, nonHPV-associated adenocarcinoma; IHC, immunohistochemistry; LVI, lymph vascular invasion; dMMR, delete mismatch repair; pMMR, proficient mismatch repair.

\section{Diagnostic performance of HPV E6/E7 mRNA in situ hybridization in ECA compared to other assays.}

In situ expression of HPV mRNA was detected using RNAscope and scored using the RNAscope scoring system. HPV mRNA was mainly detected in the cytoplasm of cancer cells, with variable staining intensity. The RNAscope scores of 0 to 4 are shown in Fig. 1A and 1B. The diagnostic implications of the HPV E6/E7 RNAscope scores were evaluated in 200 ECA samples that included cases of HPVA and NHPVA (Table S1). The positive rates of HPV PCR, p16 immunohistochemistry (IHC), HPV genotype, and HPV E6/E7 RNAscope across all cases of ECA were 76.4\%, 59.0\%, 62.5\%, and 72.0\%, respectively (Fig. $1 \mathrm{C}$ and Fig. S3). HPV RNAscope and other assays are closely related (Table S2). Receiver operating characteristics (ROC) curve analyses suggested that HPV DNA and HPV E6/E7 RNAscope showed similar results in terms of distinguishing HPVA from NHPVA (area under the curve $[A U C]=0.802$, sensitivity $=$ $80.5 \%$, specificity $=80 \%$ vs. AUC $=0.799$, sensitivity $=75.8 \%$, specificity $=80 \%$, respectively), outperforming both p16 IHC (AUC $=0.751$, sensitivity $=60.2 \%$, specificity $=90 \%)$ and HPV PCR (AUC = 0.566 , sensitivity $=63.3 \%$, specificity $=50 \%$; Fig. $1 \mathrm{D}$ and Table 5$)$.

The correlations among serum HPV DNA level, p16 IHC, HPV PCR, and HPV E6/E7 RNAscope was also studied (Table S2). A significantly higher proportion of ECA cases showed positivity for p16 IHC, HPV PCR, and HPV E6/E7 RNAscope in subgroups with HPV DNA levels that differed by 10 -fold. In patients with serum HPV levels of $1-100 \mathrm{pg} / \mathrm{mL}$, the positivity for $\mathrm{p} 16 \mathrm{IHC}$ was similar to that for HPV PCR $(60 \%)$. In patients with serum HPV levels of $100-10,000 \mathrm{pg} / \mathrm{mL}$, the positivity for HPV PCR was similar to that for HPV E6/E7 RNAscope (93.8\%). The positivity for HPV E6/E7 RNAscope for the diagnosis of ECA was much higher than that of $\mathrm{p} 16 \mathrm{IHC}$ and HPV PCR (Fig. S4A). Our results also showed that HPV E6/E7 RNAscope was positive in almost all cases that showed p16 IHC positivity ( $88.5 \%$ of all cases, $89.5 \%$ of HPVA cases), but that more cases were negative by IHC and serum levels (Fig. S4B). In different HPV subtypes, the positivity of RNAscope for the diagnosis of ECA was much higher compared to that of p16 IHC, but lower compared to HPV DNA (Fig. S4C). The HPV subtypes in patients with HPVA and NHPVA are 
listed in Table S3. In cases with RNAscope scores of 3 and 4, the positivity for HPV DNA was higher than that for p16 IHC and HPV PCR (Fig. S4D).

\section{Multivariate analysis of the OS}

Univariate analysis indicated that the following variables were related to OS in patients with ECA: age ( $p$ $=0.002)$, HPV E6/E7 RNAscope $(p=0.006)$, tumor size $(p=0.005)$, FIGO stage $(p<0.001)$, histological type $(p=0.004), L V I(p<0.001)$, invasion level of uterine cervix $(p<0.001), L N I(p<0.001)$, parametrium invasion $(p<0.001)$, surgical margin $(p=0.006)$, chemotherapy $(p=0.009)$, radiotherapy $(p<0.001)$, $p 16$ $(p=0.039)$, and $\mathrm{Ki}-67$ ( $p=0.025)$. In multivariate analysis, the following items remained independently prognostic: age $(p=0.003, H R=0.250,95 \%$ confidence interval [Cl]: 0.099-0.632), HPV E6/E7 RNAscope $(p=0.003, H R=0.240,95 \%$ Cl: 0.093-0.616), LVI ( $p=0.001, H R=1.924,95 \%$ Cl: 1.295-2.857), LNI ( $p=$ $0.021, \mathrm{HR}=3.047,95 \% \mathrm{Cl}: 1.183-7.849)$. The results are shown in Table 2, with a forest plot in Fig. 2A. Kaplan-Meier analysis showed significant diversity (Fig. 2B-E). Furthermore, the ROC curve for OS revealed that our model was higher than that of the FIGO stage and treatment (Fig. 3A).

Table 2. Univariate and multivariate Cox proportional hazards regression analysis for OS.

\begin{tabular}{|c|c|c|c|c|c|c|}
\hline \multirow[t]{2}{*}{ Variables } & \multicolumn{3}{|c|}{ Univariate analysis } & \multicolumn{3}{|c|}{ Multivariate analysis } \\
\hline & \multicolumn{2}{|c|}{ HR (95\% CI) } & \multirow[t]{2}{*}{$P$ value } & \multicolumn{2}{|c|}{ HR (95\% CI) } & \multirow{2}{*}{$\begin{array}{l}\text { Pvalue } \\
0.001\end{array}$} \\
\hline Age ( $\leq 37$ vs. $>37$ years $)$ & $\begin{array}{l}0.316 \\
0.649\end{array}$ & $(0.154-$ & & 0.224 & $(0.095-$ & \\
\hline Serum HPV DNA (negative vs. positive & 0.868 & $(0.500-$ & 0.002 & & & \\
\hline $\begin{array}{l}\text { vs. na) } \\
\text { HPV qenotype (neaative vs, positive vs, na) }\end{array}$ & $\begin{array}{l}1.509) \\
1.004\end{array}$ & (0 523- & 0.616 & & & \\
\hline & $1.926)$ & & 0.992 & & & \\
\hline HPV RNAscope ( $\leq 3.3$ vs. $>3.3$ ) & $\begin{array}{l}0.337 \\
0.730\end{array}$ & (0.156- & 0006 & 0.288 & (0.124- & 0.004 \\
\hline Tumor size $(\leq 4.5$ vs. $>4.5 \mathrm{~cm})$ & 3.165 & $(1.414-$ & 0.005 & $\begin{array}{l}0.773 \\
2.674)\end{array}$ & $(0.223-$ & 0.684 \\
\hline FIGO stage (I vs. II vs. III vs. IV) & 2.821 & $(1.703-$ & $0 \cap 00$ & 1.120 & $(0.613-$ & 0.705 \\
\hline Histological type (HPVA vs. NHPVA) & 0.266 & $(0.108-$ & .05 & 1.365 & $(0.334-$ & 0.665 \\
\hline Differentiation (good vs. moderate vs. poor) & $\begin{array}{l}1.435 \\
2.684\end{array}$ & $(0.768-$ & 0.258 & & & \\
\hline $\begin{array}{l}\text { LVI (none vs. focal vs. moderate vs. } \\
\text { extensive) }\end{array}$ & $\begin{array}{l}2.056 \\
2.803\end{array}$ & $(1.508-$ & 0.000 & $\begin{array}{l}1.770 \\
2.587)\end{array}$ & & 0.003 \\
\hline $\begin{array}{l}\text { Invasion level of uterine cervix (1/3 vs. } 1 / 3 \text { - } \\
2 / 3 \text { vs. } 2 / 3 \text { ) }\end{array}$ & $\begin{array}{l}3.147 \\
5.648\end{array}$ & (1.754- & 0.000 & $\begin{array}{l}1.532 \\
2.967)\end{array}$ & (0.791- & 0.206 \\
\hline Lymph nodes invasion (no vs. yes) & $\begin{array}{l}9.310 \\
19.12\end{array}$ & & 0.000 & $\begin{array}{l}2.838 \\
6.931)\end{array}$ & $(1.162-$ & 0.022 \\
\hline Parametrium invasion (no vs. yes) & 0.231 & $(0.094-$ & 001 & $\begin{array}{l}0.696 \\
2234)\end{array}$ & $(0.217-$ & 0.543 \\
\hline Surgical margin (no vs. yes) & $\begin{array}{l}0.263 \\
0.687\end{array}$ & (0.101- & 0.006 & $\begin{array}{l}1.621 \\
6.048)\end{array}$ & $(0.435-$ & 0.472 \\
\hline Treatment (s vs. s+ct vs. s+rt vs. s+cr) & $\begin{array}{l}1.733 \\
2.313\end{array}$ & $(1.298-$ & 0.000 & $\begin{array}{l}1.182 \\
1.641)\end{array}$ & $(0.851-$ & 0.318 \\
\hline MMR status (dMMR vs. pMMR) & $\begin{array}{l}1.401 \\
4.610\end{array}$ & $(0.426-$ & 0.579 & & & \\
\hline P16 IHC (negative vs. positive) & 0.478 & $(0.237-$ & 0039 & $\begin{array}{l}1.203 \\
2.854)\end{array}$ & $(0.507-$ & 0.675 \\
\hline Ki-67 IHC ( $\leq 12.5 \%$ vs. $>12.5 \%)$ & $\begin{array}{l}0.446 \\
0.906 \\
\end{array}$ & $(0.220-$ & 0.025 & $\begin{array}{l}0.841 \\
2.035)\end{array}$ & $(0.348-$ & 0.701 \\
\hline
\end{tabular}

HR, hazard ratio; CI, confidence interval. HPVA, HPV-associated adenocarcinoma; NHPVA, non HPVassociated adenocarcinoma; IHC, immunohistochemistry; LVI, lymph vascular invasion; dMMR, delete mismatch repair; pMMR, proficient mismatch repair; HPV genotype positive, HPV16, HPV18 and other 
types; s, surgery; s+ct, surgery+chemotherapy; s+rt, surgery+radiotherapy; s+cr, surgery+chemoradiation; na, not avaiable.

\section{Comparison of efficiency between our nomogram and conventional systems.}

A nomogram was built to predict OS. Model A included age, HPV E6/E7 RNAscope, LVI, and LNI; model B included age, HPV E6/E7 RNAscope, LVI, LNI, FIGO stage, and treatment (Fig. 3B and 3C). The results of comparison of efficiency between our nomogram and conventional systems are shown in Table 3 . In the training group, no significant difference was observed (C-index: $0.825,95 \% \mathrm{Cl}=0.754-0.896$ vs. 0.836 , $95 \% \mathrm{Cl}=0.771-0.902)$, the $\mathrm{C}$-index of nomogram A $(0.825,95 \% \mathrm{Cl}=0.754-0.896)$ was better than those of the FIGO system $(0.653,95 \% \mathrm{Cl}=0.567-0.740)$ and treatment $(0.578,95 \% \mathrm{Cl}=0.506-0.651)$. In Fig. $2 \mathrm{~A}$ and $2 \mathrm{~B}$, the calibration plot for the OS rates after 1,3, and 5-years showed agreement between the nomogram and actual observation. Besides, our model seemed to have higher prediction accuracy (Fig. 4C).

Table 3. The C-index of our model, FIGO stage, Treatment for prediction of OS in the ECA.

\begin{tabular}{llll}
\hline Factors & C-index & 95 CI\% & $P$ \\
\hline Nomogram A & 0.825 & $0.754-0.896$ & \\
FIGO stage & 0.653 & $0.567-0.740$ & \\
Treatment & 0.578 & $0.506-0.651$ & \\
Nomogram B & 0.836 & $0.771-0.902$ & 0.002 \\
Nomogram A vs FIGO stage & & & $<0.001$ \\
Nomogram A vs Treatment & & & 0.139 \\
\hline
\end{tabular}

Nomogram A: age + HPV RNA scope + LVI + Lymph nodes invasion Nomogram B: age + HPV RNA scope + LVI + Lymph nodes invasion+ FIGO stage+ Treatment C-index = concordance index; LVI, lymph vascular invasion; $\mathrm{P}$ values are calculated based on normal approximation using function rcorrp.cens in Hmisc package.

\section{Risk stratification of prognosis}

All patients were divided into a low-risk group ( $\leq 15.75)$ and a high-risk group (>15.75) for OS (Fig. S2B). The 1-, 3-, and 5-year OS rates between the two risk groups were $94.2 \%, 84.3 \%, 70.3 \%$, respectively, in the low-risk group and $71.4 \%, 46.4 \%, 10.7 \%$, respectively, in the high-risk group (Table 4). Moreover, significant differences in OS were noted between patients with stage I/II ECA and those with III/IV ECA (Fig. 5). Each risk subgroup represented a distinct prognosis, and our system accurately separated OS in the two subgroups. 
Table 4. Point Assignment and Prognostic Score of the nomogram model

\section{Variables}

\section{Prognostic Score 1-Year OS (\%)}

3-Year OS (\%) 5-Year OS (\%)

\begin{tabular}{lllll}
\hline Age (years) & & & \\
\hline$\leq 37$ & 7.5 & 11.8 & 26.5 & 35.3 \\
\hline$>37$ & 0 & 1.8 & 6.0 & 10.8 \\
\hline HPV RNA scope & 7 & 5.1 & 13.1 & 21.2 \\
\hline$\leq 3.3$ & 0 & 2.0 & 5.9 & 8.9 \\
\hline$>3.3$ & & & 4.5 & 7.1 \\
\hline Lymph nodes invasion & 0 & 1.9 & 26.1 & 41.3 \\
\hline No & 8.25 & 8.7 & & 10.1 \\
Yes & & & 6.5 & 13.5 \\
Lymph vascular invasion & 0 & 1.4 & 10.8 & 26.7 \\
None & 3.25 & 2.7 & 20.0 & 70.0 \\
Focal & 6.5 & 13.3 & 30.0 & 70.3 \\
Moderate & 9.75 & 20.0 & & 10.7 \\
\hline Extensive & & & 84.3 & \\
\hline Total prognostic Score & & 94.2 & 46.4 & \\
\hline$\leq 15.75$ & & 71.4 & & \\
\hline$>15.75$ & & & & \\
\hline
\end{tabular}

Table 5. Diagnostic performances of studied testing for ECA patients.

\begin{tabular}{lllll}
\hline HPVA vS. NHPVA & AUC & 95\%CI & Sensitivity & Specificity \\
\hline P16 IHC & 0.751 & $0.620-0.882$ & 0.602 & 0.900 \\
HPV DNA & 0.802 & $0.653-0.951$ & 0.805 & 0.800 \\
\hline HPV genotype & 0.566 & $0.379-0.754$ & 0.633 & 0.500 \\
HPV RNAscope & 0.799 & $0.629-0.929$ & 0.758 & 0.800 \\
\hline
\end{tabular}

AUC, area under curve; 95\%CI, 95\% confident interval; HPVA, HPV-associated adenocarcinoma; NHPVA, nonHPV-associated adenocarcinoma; IHC, immunohistochemistry; HPV RNAscope.

\section{Discussion}

The present study was the first to describe a prognostic nomogram model for invasive ECA in a large Chinese series, reporting HPV E6/E7 RNAscope using a new RNA ISH assay that recognizes 18 high-risk HPV types. The most important results were as follows: (1) the validity of the HPVA and NHPVA categories were supported by p16 immunophenotyping and HPV status; (2) the high-risk HPV probe set is more sensitive and specific than p16, HPV DNA PCR, and HPV genotype for recognizing HPVA; (3) we established a prognostic nomogram model based on pathogenesis that included age, LVI, HPV E6/E7 RNAscope, and LNI, and the model had greater predictive efficiency than the current conventional staging system; (4) based on our model, patients could be divided into low-risk and high-risk groups.

HPV E6/E7 RNAscope measurement is not routinely available in most hospitals and the methodology is not globally standardized. In this regard, our findings suggest that clinicians should increase HPV E6/E7 RNAscope measurement in clinical practice. About $94 \%$ of adenocarcinomas are associated with human papillomavirus (HPV), particularly with strains 16 and 18(5), consistent with our results (Table S3). In the present study, 87\% (160/184) of HPVA samples overexpressed p16 or were HPV + by RNAscope, 
validating the IECC criteria and consistent with a previous study $(9,22)$. In terms of the IECC criteria, the HPV RNAscope is more specific for clinical practice, and it is more robust than p16 IHC, HPV PCR, and HPV genotype to identify HPVA. Despite this, occasional p16 and/or HPV- HPVAs were identified. When outlying cases were excluded from statistical analysis, 25 of the 184 HPVA cases were p16- and HPVpositive. All HPVA cases in the present study were confirmed on review. The specimens were more than 5 years, may not optimal tissues for the performance of HPV RNAscope and p16. A recent study reported that p16- and HPV-positive, usual-type ECA might represent unusual morphological variants of gastrictype carcinoma(23). Furthermore, rare HPV genotypes not included in the RNA ISH probe set may be responsible for negative HPV results. There are precedents in the literature for $\mathrm{p} 16$ - and HPV-associated neoplasia. Specifically, p16-positive invasive squamous carcinomas of the cervix have been reported(24), as has methylation-induced inactivation of the $p 16$ gene and allelic loss of $p 16(25,26)$. Other studies have reported that the HPV genome can be differentially expressed between primary tumors and metastases, as well as among different disease sites, suggesting that some "HPV-related," usual-type adenocarcinomas may have had detectable HPV in multiple sections from the primary carcinoma and that all metastatic sites should been tested(22, 27, 28).

Clinicopathological variables have prognostic significance and presently impact clinical management(29, 30). Depending on variables such as LVI and desire for fertility may be considered(31). Depending on variables such as LVI and desire for fertility may be considered(32), so histological evaluation of risk would be beneficial at the time cervical samples are taken. The National Comprehensive Cancer Network guidelines recommend chemotherapy or radiotherapy as the standard treatment for patients with advanced ECA in clinical practice. Hence, the performance of nomograms must be examined separately in patients treated using these therapies. The $\mathrm{C}$-indices of nomograms $\mathrm{A}$ and $\mathrm{B}$ for predicting $\mathrm{OS}$ in treated patients were significantly higher than those of conventional classification, indicating that nomograms still have significant clinical value in patients with ECA treated using chemotherapy or radiotherapy. With the addition of the FIGO stage and treatment into nomogram $B$, the added values of these parameters over nomogram A were 0.836 and 0.825 , respectively, across the entire population. There were no significant differences between nomograms A and B, probably because the number of patients with advanced-stage ECA was small.

The present study had several shortcomings. Firstly, there may have been selection bias. Secondly, we lacked data on the impact of our nomogram on other prognoses, such as disease-free survival (DFS) prediction. Thirdly, the in situ measurement of HPV RNA using RNAscope must still be globally standardized. Fourthly, our sample size was relatively small and collected from one center. To verify our results, more samples should be collected from other institutes. Lastly, it remains unclear whether our nomogram can be applied to advanced-stage patients (stages III and IV).

In summary, we built a portentous nomogram for prognosis in the patients with ECA. It was more accurate than the conventional FIGO staging system. The model is simple, economical, and helpful for clinical decision making. 


\section{Conclusions}

We generated new nomograms incorporating HPV E6/E7 MRNA in situ hybridization and clinical characteristics to prognose the OS rate in patients with ECA. Our simple and explicit nomograms have good clinical application value, and they show good discrimination and calibration ability. They may be a useful tool for assessing prognosis and managing treatment in patients with ECA.

\section{Abbreviations}

HPV: Human papillomavirus; HPV E6/E7 RNAscope: Human papillomavirus E6/E7 mRNA in situ hybridization; ECA: Endocervical adenocarcinoma; C-index: Concordance index; HPVA: HPV-associated adenocarcinoma; NHPVA: non HPV-associated adenocarcinoma; OS: Overall survival; LVI: Lymphovascular invasion; LNI: Lymph node involvement.

\section{Declarations}

\section{Ethics approval and consent to participate}

The Sun Yat-sen University Cancer Center Ethics Committee approved this study.

\section{Consent for publication}

Not applicable.

\section{Availability of data and materials}

The authenticity of this article has been validated by uploading the key raw data onto the Research Data Deposit public platform (www.researchdata.org.cn) with the approval RDD number RDDA2020001638.

\section{Competing interests}

The authors declare that they have no competing interests.

\section{Funding}

This work was supported by the National Natural Science Foundation of China (No. 81872012, 81872266).

\section{Authors' contributions}

Conception and design: Ting Wang and Rong-Zhen Luo. Performing experiments: Li-Li Liu, Shu-Mei Yan and Xia Yang. Drafting of the article: Li-Li Liu and Shu-Lin Chen. Acquisition and interpretation of data, review, editing and approval of the manuscript: all authors.

\section{Acknowledgements}


The authors gratefully acknowledge the assistance from professor Jing-Ping Yun from Sun Yat-sen University Cancer Center.

\section{References}

1. Ward KK, et al. (2012) Changing demographics of cervical cancer in the United States (1973-2008). Gynecol Oncol 126: 330-333.

2. Galic V, et al. (2012) Prognostic significance of adenocarcinoma histology in women with cervical cancer. Gynecol Oncol 125: 287-291.

3. Bhatla N, et al. (2019) Revised FIGO staging for carcinoma of the cervix uteri. Int J Gynaecol Obstet 145: 129-135.

4. Stolnicu S, et al. (2019) Clinical Outcomes of HPV-associated and Unassociated Endocervical Adenocarcinomas Categorized by the International Endocervical Adenocarcinoma Criteria and Classification (IECC). Am J Surg Pathol 43: 466-474.

5. RJ K, ML C, CS H, RH Y. (2014) WHO Classification of Tumors of Female Reproductive Organs, 4th ed. Lyon: IARC, WHO Press.

6. Byun JM, et al. (2019) Clinical significance of the pattern-based classification in endocervical adenocarcinoma, usual and variants. Int J Clin Oncol 24: 1264-1272.

7. Karamurzin YS, et al. (2015) Gastric-type Endocervical Adenocarcinoma: An Aggressive Tumor With Unusual Metastatic Patterns and Poor Prognosis. Am J Surg Patho/ 39: 1449-1457.

8. Stolnicu S, et al. (2018) Stromal invasion pattern identifies patients at lowest risk of lymph node metastasis in HPV-associated endocervical adenocarcinomas, but is irrelevant in adenocarcinomas unassociated with HPV. Gynecol Oncol 150: 56-60.

9. Stolnicu S, et al. (2018) International Endocervical Adenocarcinoma Criteria and Classification (IECC): A New Pathogenetic Classification for Invasive Adenocarcinomas of the Endocervix. Am J Surg Pathol 42: 214-226.

10. Cheung TH, et al. (2019) Liquid biopsy of HPV DNA in cervical cancer. J Clin Virol 114: 32-36.

11. McCluggage WG, Jenkins D. (2003) p16 immunoreactivity may assist in the distinction between endometrial and endocervical adenocarcinoma. Int J Gynecol Pathol 22: 231-235.

12. Kenemans P. (1994) HPV genotype as a prognostic factor for progression to cervical carcinoma in young women. Eur J Obstet Gynecol Reprod Biol 55: 24-25.

13. Schache AG, et al. (2013) Validation of a novel diagnostic standard in HPV-positive oropharyngeal squamous cell carcinoma. Br J Cancer 108: 1332-1339.

14. Mirghani H, et al. (2015) Diagnosis of HPV-driven head and neck cancer with a single test in routine clinical practice. Mod Patho/ 28: 1518-1527.

15. Roma AA, et al. (2017) Role of Lymphovascular Invasion in Pattern C Invasive Endocervical Adenocarcinoma. Am J Surg Pathol 41: 1205-1211. 
16. Sathish N, et al. (2004) HPV DNA in plasma of patients with cervical carcinoma. J Clin Virol 31: 204209.

17. Chera BS, et al. (2020) Plasma Circulating Tumor HPV DNA for the Surveillance of Cancer Recurrence in HPV-Associated Oropharyngeal Cancer. J Clin Oncol 38: 1050-1058.

18. Hodgson A, et al. (2019) Genomic Characterization of HPV-related and Gastric-type Endocervical Adenocarcinoma: Correlation With Subtype and Clinical Behavior. Int J Gynecol Pathol.

19. Anderson CM, et al. (2016) Fully Automated RNAscope In Situ Hybridization Assays for FormalinFixed Paraffin-Embedded Cells and Tissues. J Cell Biochem 117: 2201-2208.

20. Bakheet $\mathrm{AMH}$, et al. (2020) Improving pathological early diagnosis and differential biomarker value for hepatocellular carcinoma via RNAscope technology. Hepatol Int 14: 96-104.

21. Camp RL, Dolled-Filhart M, Rimm DL. (2004) X-tile: a new bio-informatics tool for biomarker assessment and outcome-based cut-point optimization. Clin Cancer Res 10: 7252-7259.

22. Molijn A, et al. (2016) The complex relationship between human papillomavirus and cervical adenocarcinoma. Int $J$ Cancer 138: 409-416.

23. Wada T, et al. (2017) Endocervical Adenocarcinoma With Morphologic Features of Both Usual and Gastric Types: Clinicopathologic and Immunohistochemical Analyses and High-risk HPV Detection by In Situ Hybridization. Am J Surg Patho/ 41: 696-705.

24. Masoudi $\mathrm{H}$, et al. (2006) Loss of p16 INK4 expression in invasive squamous cell carcinoma of the uterine cervix is an adverse prognostic marker. Histopathology 49: 542-545.

25. Nuovo GJ, Plaia TW, Belinsky SA, Baylin SB, Herman JG. (1999) In situ detection of the hypermethylation-induced inactivation of the p16 gene as an early event in oncogenesis. Proc Natl Acad Sci U S A 96: 12754-12759.

26. Poetsch M, et al. (2011) Alterations in the tumor suppressor gene p16(INK4A) are associated with aggressive behavior of penile carcinomas. Virchows Arch 458: 221-229.

27. Beyer-Finkler E, Girardi F, Sillem M, Pfister H. (1995) Human papillomavirus DNA in genital cancers, metastases, and lymph nodes. Intervirology 38: 173-180.

28. Liang WS, et al. (2014) Simultaneous characterization of somatic events and HPV-18 integration in a metastatic cervical carcinoma patient using DNA and RNA sequencing. Int J Gynecol Cancer 24: 329338.

29. Ronnett BM. (2016) Endocervical adenocarcinoma: selected diagnostic challenges. Mod Pathol 29 Suppl 1: S12-28.

30. Baalbergen A, Ewing-Graham PC, Hop WC, Struijk P, Helmerhorst TJ. (2004) Prognostic factors in adenocarcinoma of the uterine cervix. Gynecol Oncol 92: 262-267.

31. Pecorelli S. (2009) Revised FIGO staging for carcinoma of the vulva, cervix, and endometrium. Int J Gynaecol Obstet 105: 103-104.

32. Diaz De Vivar A, et al. (2013) Invasive endocervical adenocarcinoma: proposal for a new patternbased classification system with significant clinical implications: a multi-institutional study. Int $J$ 
Gynecol Patho/ 32: 592-601.

Figures
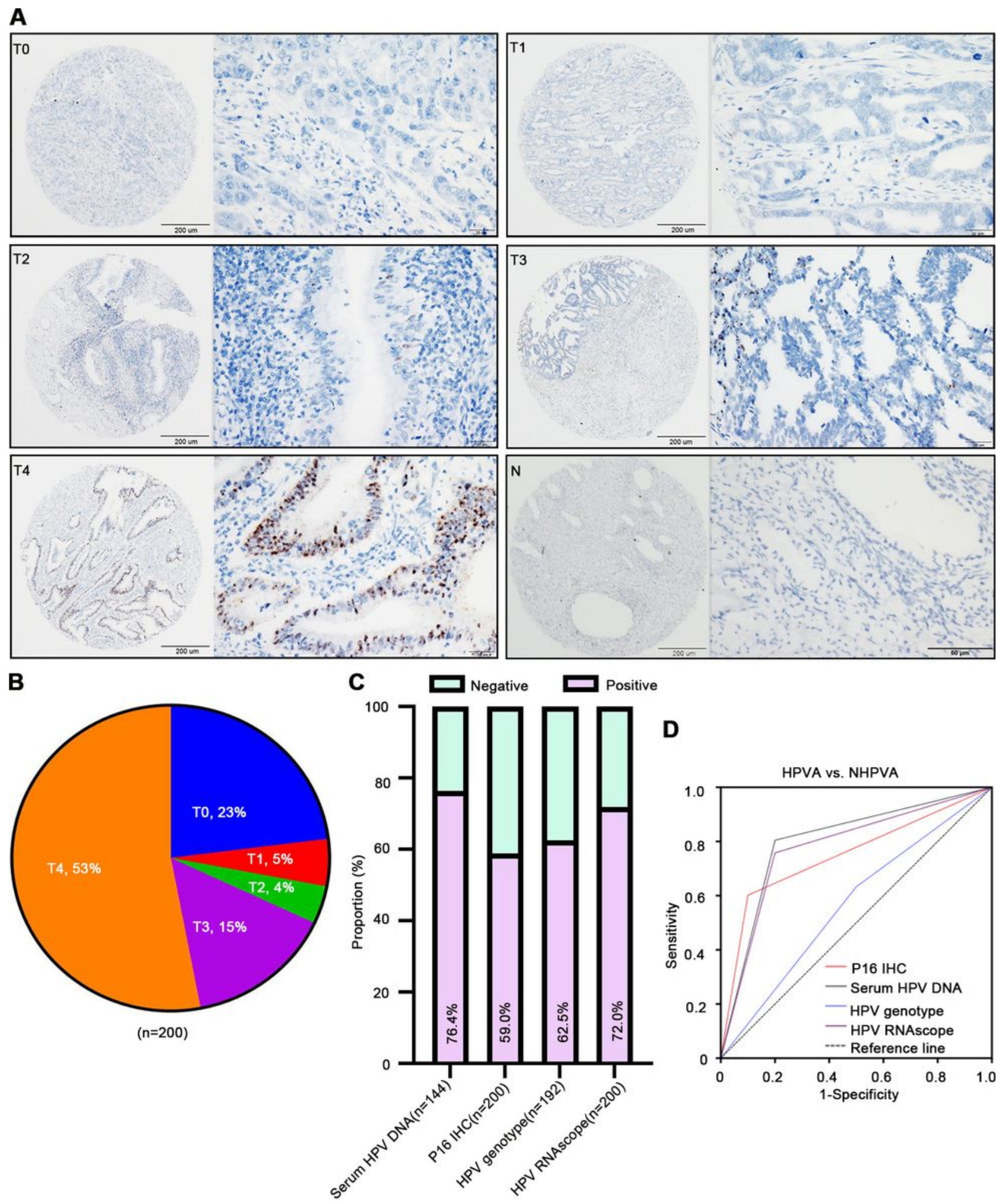

Figure 1 
Representative images of human papillomavirus (HPV) E6/E7 RNAscope in paraffin-embedded endocervical adenocarcinoma (ECA) samples. (A) HPV mRNA was detected using RNAscope in ECA (T) and non-tumorous tissues adjacent to ECA $(\mathrm{N})$. Representative images of scores 0 (T0), 1 (T1), 2 (T2), 3 (T3), and 4 (T4) are shown. (B) Proportion of RNAscope scores in ECA tissues. (C) Positive rates of HPV by PCR, RNAscope, HPV genotype, and p16 IHC. (D) Receiver operating characteristics curve for HPV, detected by PCR, immunohistochemistry, genotyping, and RNAscope for HPV-associated vs. non-HPVassociated types.

A

Variables Pvalue Hazard Ratio $(95 \% \mathrm{Cl})$

Age $\quad 0.003 \quad 0.250(0.0999 .0 .632)<--1$

HPV RNAscope $\quad 0.003 \quad 0.240(0.093-0.616)<-\cdots-\cdots$

LVI $\quad 0.001 \quad 1.924(1.295-2.857)$

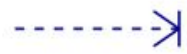

LNI $\quad 0.021 \quad 3.047(1.183 .7 .849)$
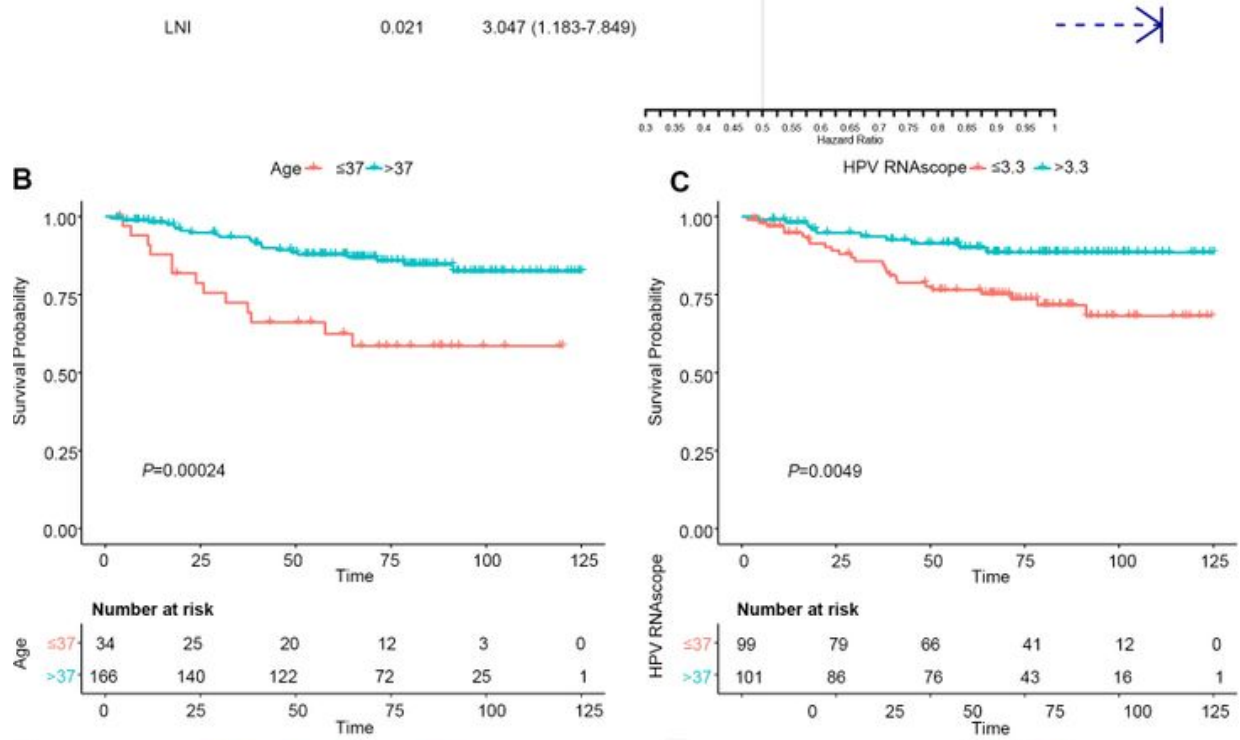

D

E
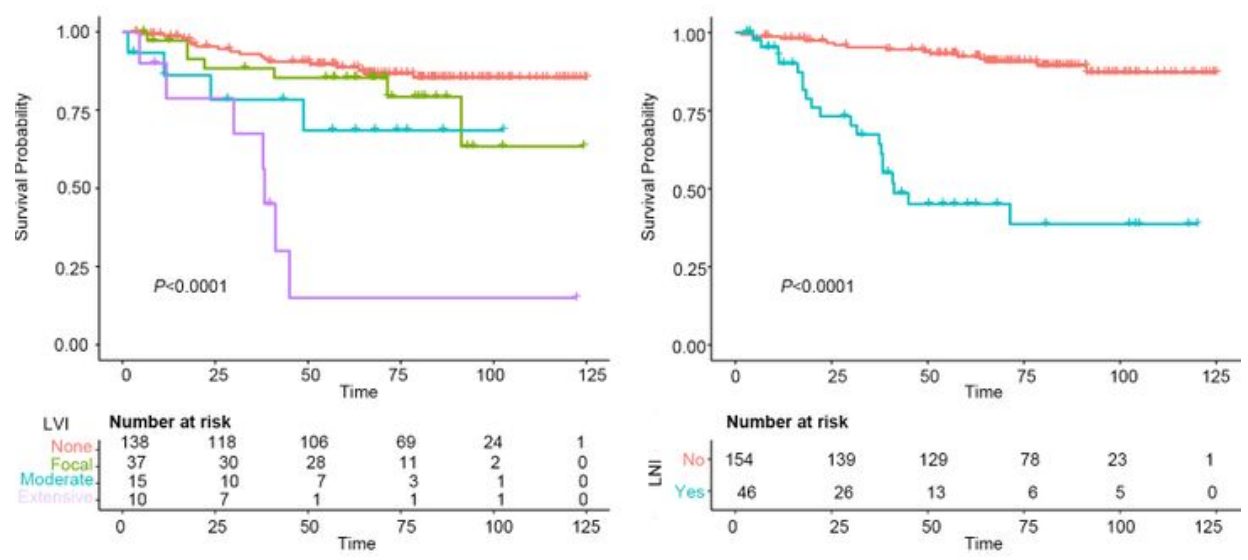
Figure 2

Forest plot and Kaplan-Meier curves for overall survival (OS) in patients with endocervical adenocarcinoma (ECA). (A) Forest plot showed the hazard ratio and $95 \%$ confidence interval for OS according to the Cox proportional hazards regression analysis in patients with ECA. (B-E) Age, human papillomavirus E6/E7 RNAscope, lymphovascular invasion, and lymph node involvement in patients with ECA in whole cohort are plotted as a distribution.

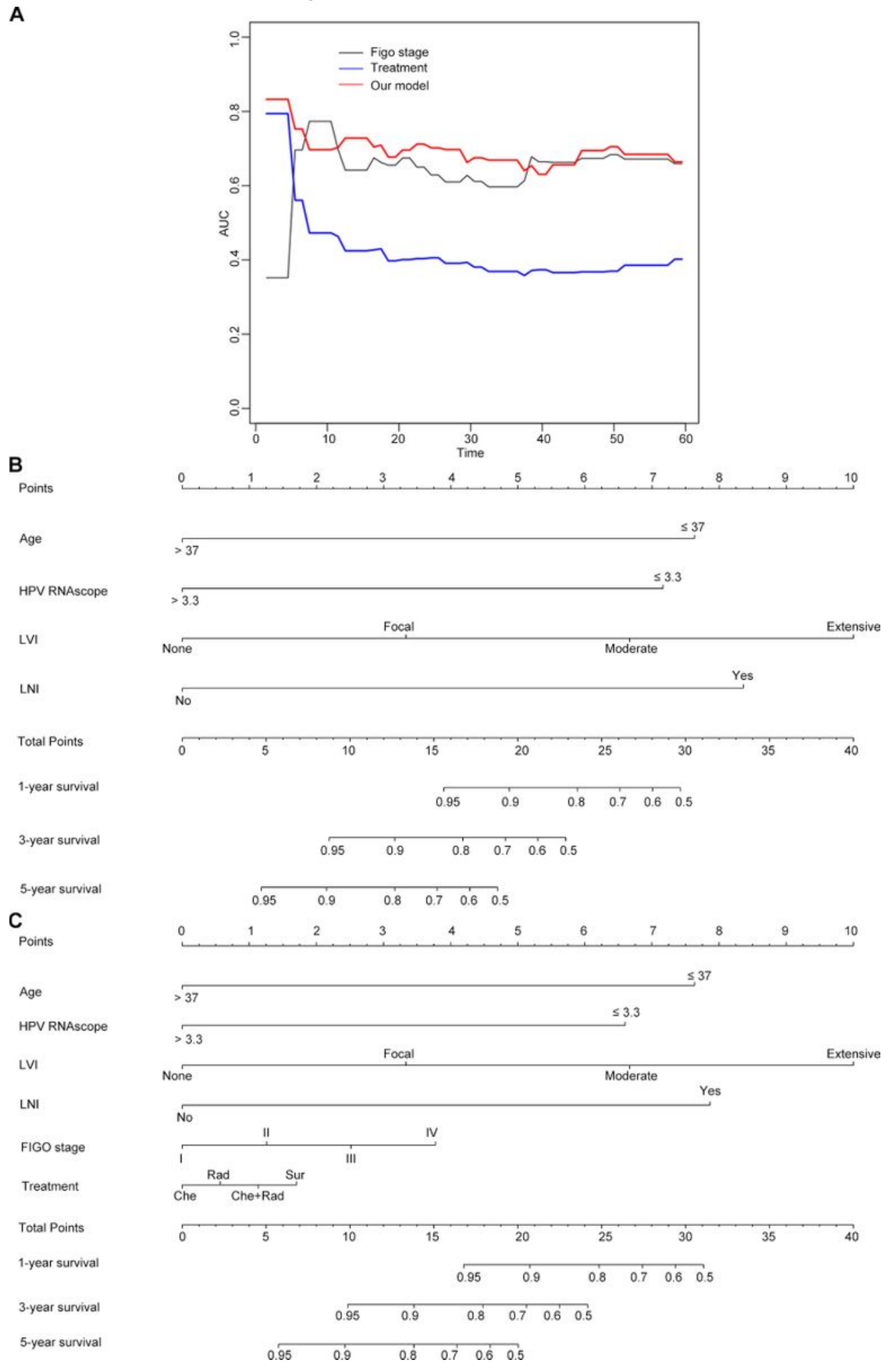


Figure 3

Time-dependent ROC curve analysis and nomogram model for overall survival (OS) in patients with endocervical adenocarcinoma ECA. (A) Time-dependent receiver operating characteristic curves showing the sensitivity and specificity of the nomogram model for predicting OS. (B-C) The nomogram models $A$ and $B$ were used to summate the points identified on the points scale for each variable, indicating the probability of 1-, 3-, and 5-year survival.
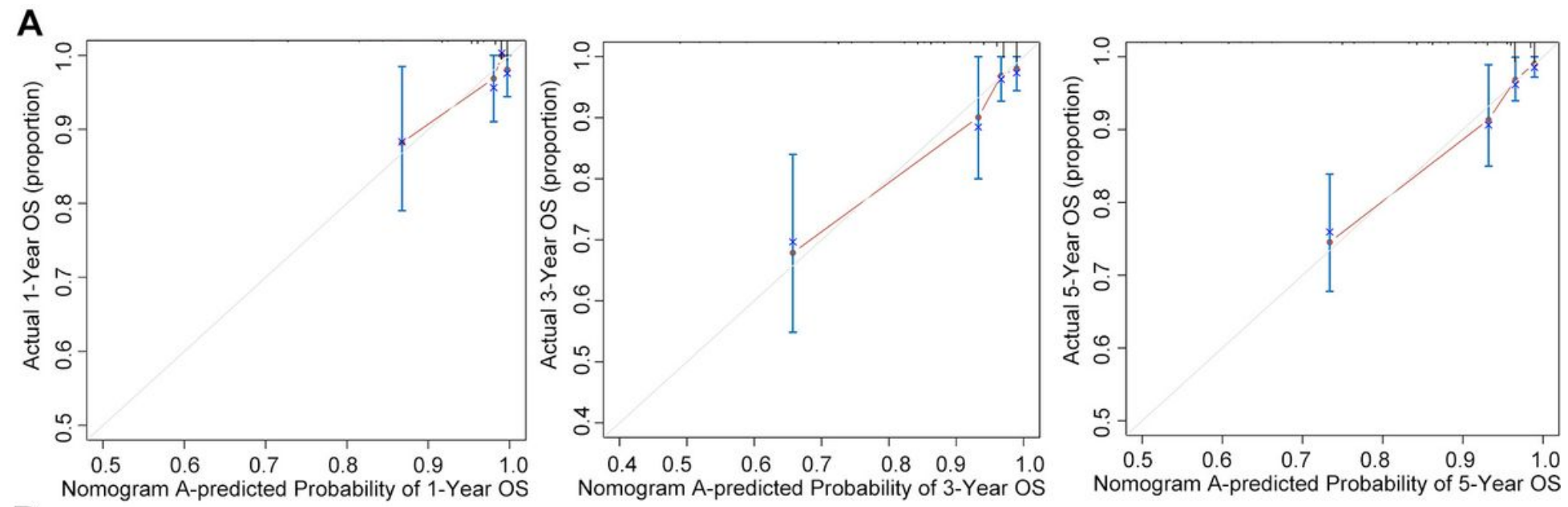

B
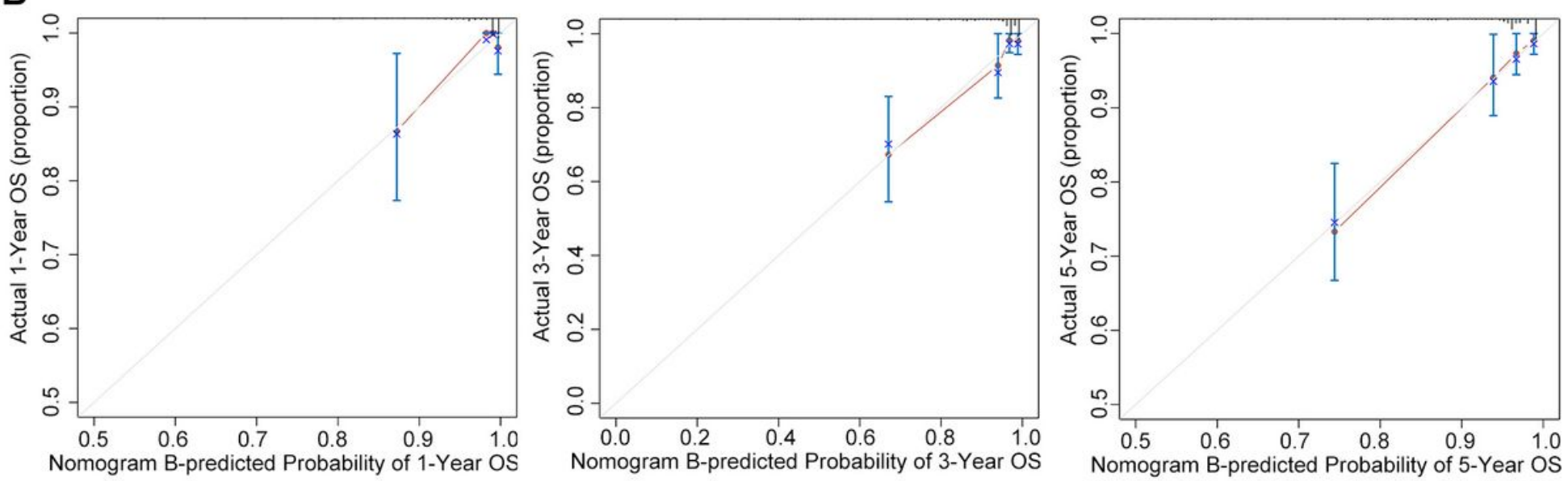

C
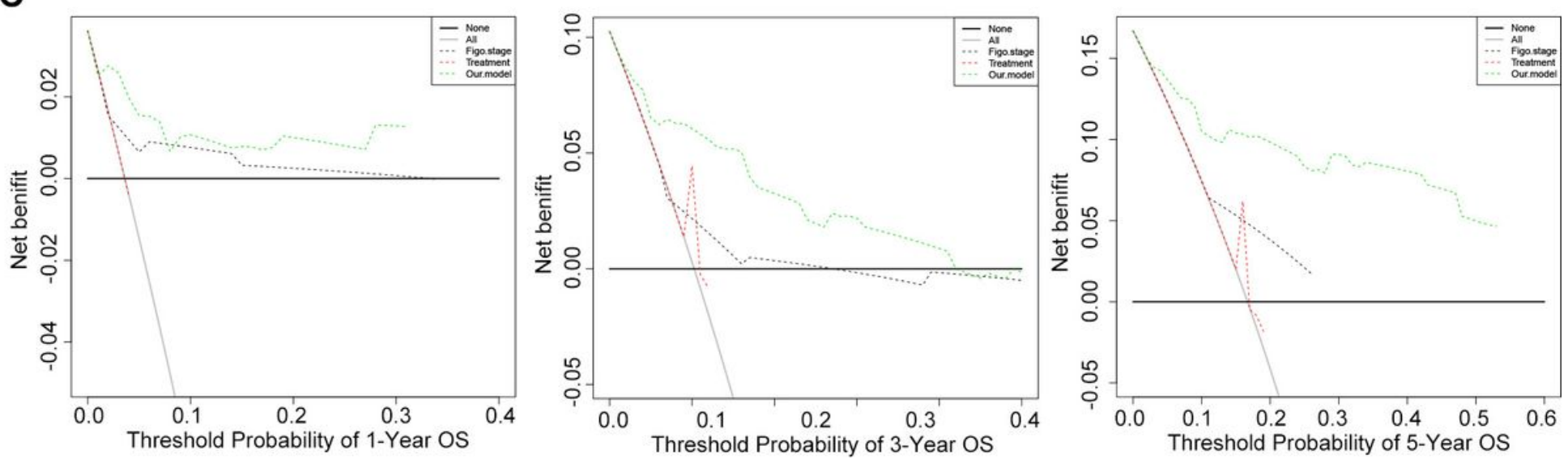

Figure 4 
The calibration curves, decision curve analysis for predicting patient overall survival (OS). The calibration curves for predicting OS at 1, 3, and 5 years in all cases. (A\&B) Nomograms A and B, with model-predicted OS plotted on the $x$-axis and actual OS plotted on the $y$-axis. Closer alignment with the diagonal line represents better estimation. (C) Decision curve analysis for 1-, 3-, and 5-year survival predictions.
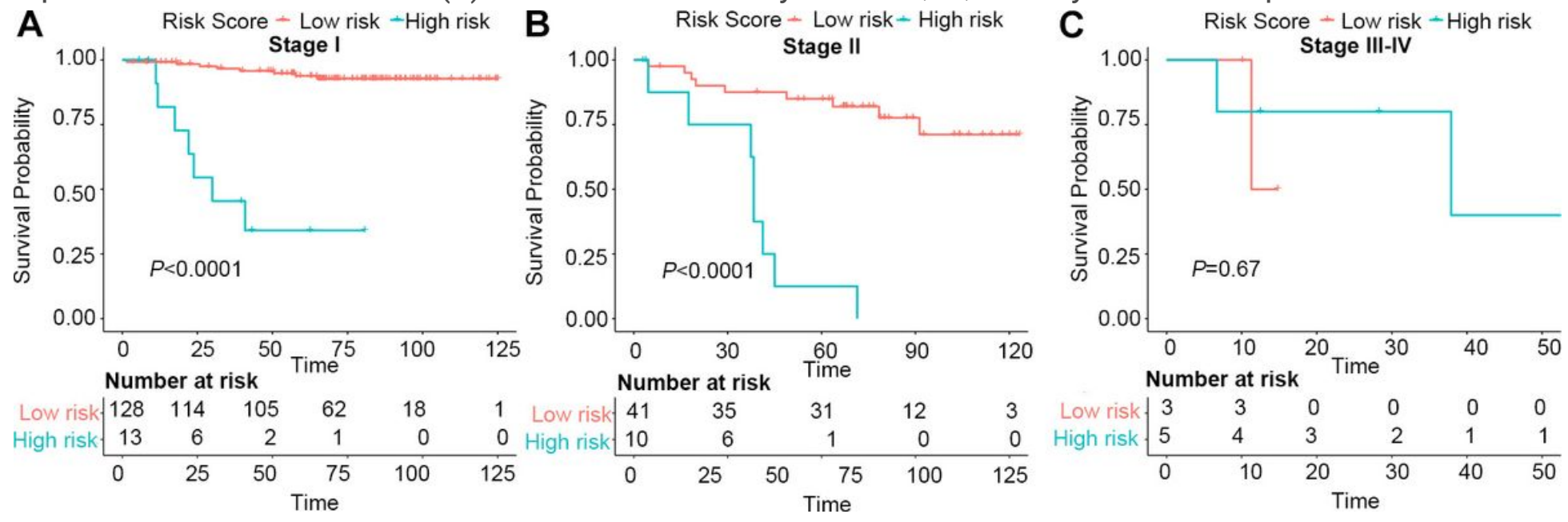

Figure 5

Graphs showing the Kaplan-Meier curves of both groups based on the predictors from the nomogram model. (A) stage I. (B) stage II. (C) stages III and IV.

\section{Supplementary Files}

This is a list of supplementary files associated with this preprint. Click to download.

- SupplementalTables.docx

- SupplementalFigures.docx 